PRODUCTION

ENGINEERING ARCHIVES
2016, Vol. 10, No 1, pp 13-16

ISSN 2353-5156 (print version)

ISSN 2353-7779 (online version)

\title{
Software support for environmental measurement in quality at educational institutions
}

\author{
Alena Pauliková ${ }^{1}$, Katarína Čekanová $^{1}$, Renata Nováková $^{1}$ \\ ${ }^{1}$ SlovakUniversity of Technology in Bratislava, Faculty of Materials Science and Technology in Trnava, Institute of safety, environment and \\ quality, Botanická 49, 91724 Trnava, Slovakia, phone: 00421/0908646519, e-mail: katarina.cekanova@stuba.skalena.paulikova@stuba.sk,
}

\begin{abstract}
The analysed theme of this article is based on the training of environmental measurements for workplaces. This is very important for sustainable quality in technical educational institutions. Applied kinds of software, which are taught at technical educational institutions, have to offer the professional and methodical knowledge concerning conditions of working ambient for students of selected technical specialisations. This skill is performed in such a way that the graduates, after entering the practical professional life, will be able to participate in solutions for actual problems that are related to environmental protection by means of software support. Nowadays, during the training process it is also obligatory to introduce technical science. Taking into consideration the above-mentioned facts it is possible to say that information technology support for environmental study subjects is a relevant aspect, which should be integrated into the university educational process. There is an effective progress that further highlights the focus on the quality of university education not only for environmental engineers. Actual trends require an increasing number of software/hardware educated engineers who can participate in qualitative university preparation, i.e.IT environmentalists. The Department of Environmental Engineering at the Faculty of Mechanical Engineering, Technical University in Košice, Slovakia is an institution specified and intended for quality objectivisation. This institution introduced into the study programmes ("Environmental Management" and "Technology of Environmental Protection") study subjects with the software support, which are oriented towards outdoor and indoor ambient and in this way the Department of Process and Environmental Engineering is integrated effectively and intensively into the area of measurement training with regard to the requirement of quality educational processes.
\end{abstract}

Key words -software support, indoor, outdoor, measurement, quality

\section{Introduction}

Nowadays there is a frequently repeated idea that information technology support has a key role in environmental education as well as skills in measurement performance and expresses an irreplaceable function in the field of sustainable development. At the technical educational institutions there is the important intent of software education to provide the professional and methodical knowledge about IT skills for students. It is presented in such a way that in practice they can participate in seeking a solution for challenging specific problems which are closely related to environmental measurement. At present, in the educational process there is sn obligation to introduce technical development to an increasingly high standard (CHOVANCOVÁ J., HARAUSOVÁ H. 2013).

\section{Software support for environmental measurement}

The selected institution for environmental objectivisation was the department of Process and Environmental Engineering, Mechanical Engineering Faculty, 
Technical University in Košice, the Slovak Republic. This institution demonstrates the synergistic effect of information technology skills and environmental education within the framework of the strengthening of quality development (MARKULIK, Š., NAGYOVÁ, A. 2008).

\section{Measuring of noise and vibration}

For measurement of noise and vibration the phonometer Norsonic 121 and Norsonic 140 (see Fig. 1) are used.

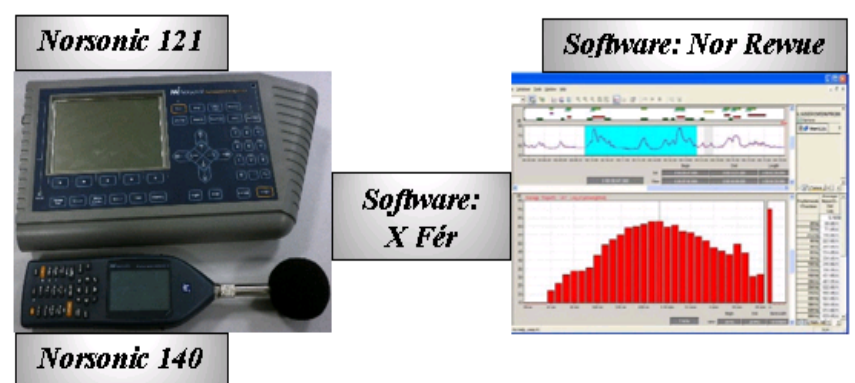

Fig. 1. Scheme of the measured noise data transferring from the phonometer to PC (Source: own study)

Source: own study

Apparatus perform creation of the sound record, evaluation of the structural acoustics, identification of the sound source, creation of the sound maps, noise measuring in outdoor and indoor environments, measuring of the vibrations (with completing of the software evaluated modem). There are characteristics, more detailed descriptions of the apparatus and its purpose of the important elements.

Software X Fér connects the data transfer from the phonometer to the PC. It secures the transfer of the measured data together with necessary explaining data from the phonometer.

Software Nor Rewue is designed for processing of the sound and vibration data. It is used for the creation of simple reports and eventually to the creation of more widening developing and complex projector reports. It serves for evaluation of industry and traffic noise.

The acoustic camera Array-Ring 32/75 and acoustic camera Array-Ring 36/48 are other devices which are used for the noise measuring by students during their studies. The acoustic camera is an apparatus designed for the visualisation, localization and analysis of the sources of the noise. It is suitable for the measuring of the vast industry sources of the noise and also for measuring very quiet sounds in the laboratories for purposes of identification and the following quantitative and qualitative analyse. The scheme of the apparatus arrangement represents in Fig.2.

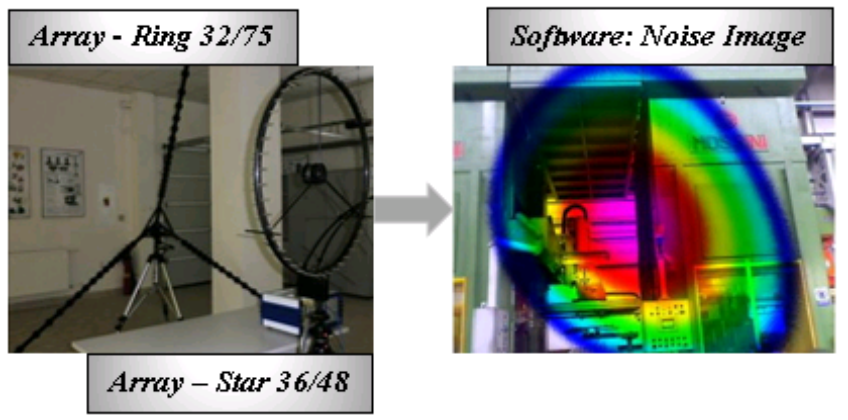

Fig. 2. Scheme of the apparatus arrangement for noise measuring with the acoustic camera (Source: own study)

Source: own study

Software Noise Image enables the processing of measured data from the acoustic camera. It enables the transfer into the evaluating equipment, the evaluation of these data, the creation of the acoustic snaps and films shots. Noise Image is developed with the purpose of the reaching the interaction among the space, time and frequency. At the model creation there is observed only the equivalent level of the acoustic pressure. In the acoustic picture the values colour differed in such a way that they could be created with the point sources.

The apparatus performs the following operations:

- the spectral analyse of the pictures

- $\quad$ the data edition for individual channel

- the narrow-band filtration

- the utilization of the built-in oscilloscope for the definition of the time and spectral functions

- the linear and the logarithmic A evaluation.

The materials absorption and isolation is measured by means of a device called the Impedance tube $S W$ 466, Fig 3.

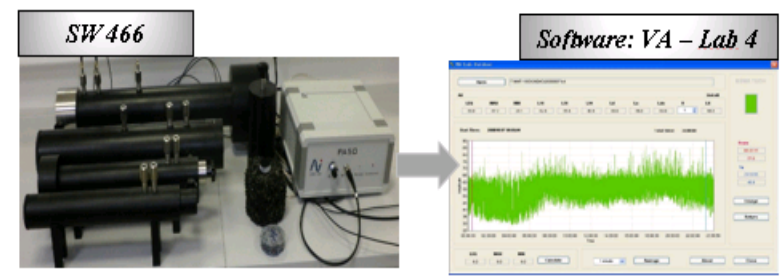

Fig. 3. The set up scheme of an apparatus and outputs of material absorption measurements,(Source: own study)

Source: own study 
In the laboratory the psychoacoustic head HSU III is used for measuring of psychoacoustic noise. This apparatus analyses the influence of noise on human psychic and operates on the basis of the statistical data. It is able to analyse the noise perceptions as effectively as a human ear. It evaluates the physical and psychoacoustic parameters of the noise for human psychic.

The program VA-Lab4 performs analysis which is necessary for noise measuring. The obtained values appear in a different time period. The program is effective to create a curve with selected average times (CRYSTAL INSTRUMENTS 2009).

Vibrations are an attendant factor of the noise. These parameters are measured in the laboratory with the apparatus CoCo 80, Fig. 4.

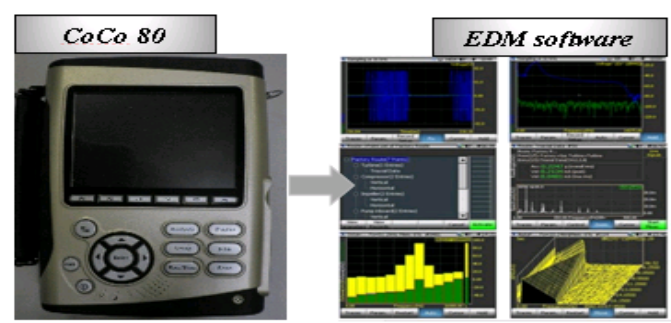

Fig. 4. The set up scheme and software outputs of the vibration measurements (Source: own study)

Source: own study

In principle the device CoCo 80 is a vibration analyser dedicated to measuring in an industrial environment. EDM software creates the protocols and diagrams and their dependences from the device Crystal Instrument CoCo 80 which supports the registration of measured results (TESTO 400 A 435 2009).

\section{Illumination}

The illumination is a further factor which significantly influences the quality of the working environment. The analysis of this factor is also a basic part of the environmental engineers' knowledge. The students learn to operate with Luxmeter Testo 545 and Radiolux 111, Fig.5.

Luxmeter Testo 545 is designed for the measuring of the illumination intensity in the producing workplaces, in the institutions and schools. By means of the program it is possible to report the list of the measuring points and then to generate a graph from consecutive loaded values. In such a way created ,light profile" gives a review of the illumination equability of the evaluated environment.
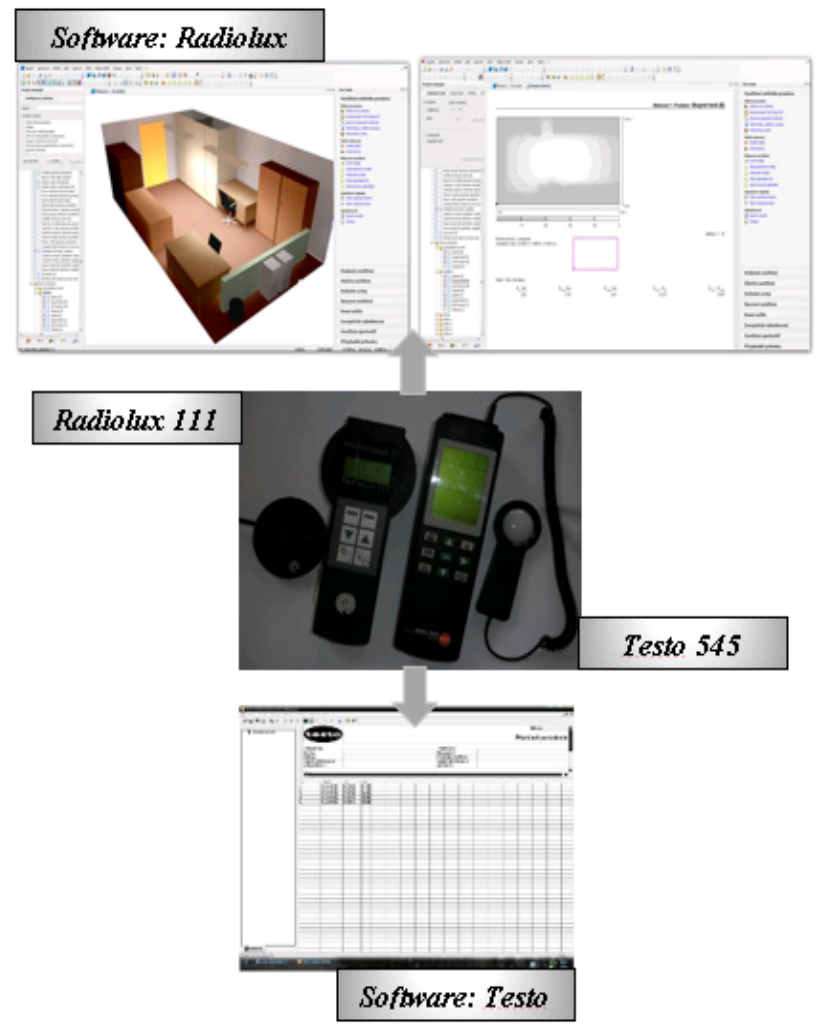

Fig. 5. The scheme and the software outputs for the illumination measurement (Source: own study)

Source: own study

The luxmeter has got the following functions designed for the illumination intensity evaluation:

the determination of the point or time medium value:

- the selection of more than 99 measuring points

- inner memory (3000 values)

- possibility of the rapid documentation by means of the protocol printer Testo in place, (optional)

Radio Lux 111 is a manual portable apparatus designed for photometric and radiometric measurement and enables light measuring and the light radiation. It is suitable for external field measurements for accurate laboratory measurement as well. The brightness measurement is a supplementary function which can be accessible with a special adapter.

Software is an integral part of Luxmeter Testo 545 and Radiolux 111 and creates the interface for data transferring from the luxmeters to the PC (TESTO 545 2009).

\section{Electromagnetic radiation}


Electromagnetic radiation is measured by the apparatus Efa 200. The device is an analyzer which is designed for the magnetic and electric field measuring of the indoor and outdoor environment. The apparatus works with the software Efa tool and software NBMTS. Efa tool is designed for the gathering and transferring of the measured data from devices (control unit of the apparatus Efa 200/300) to the PC. It enables the outputs in the rtf format.

Software NBM-TS provides the visualisation, analysis of the measured results and their exporting the table calculator MS Excel (NARDA SAFETY TEST SOLUTIONS 2009).

\section{Microclimate conditions}

The evaluation of the basic physical environmental factors also represents the measuring of the microclimate conditions. Microclimate is measured by means of the apparatus Testo 435 and Testo 400. The device Testo 435 enables the measuring of the following parameters of the air and environment:

- temperature - turbulence

- relative humidity - differential pressure

- illumination - relative pressure

(NARDA SAFETY TEST

SOLUTIONS 2009)

- speed of airflow

The apparatus Testo 400 is as the reference measuring equipment enabling the large scale measuring of the environment parameters, gas, liquid and rigid matters as well. This device performs measurements for following:

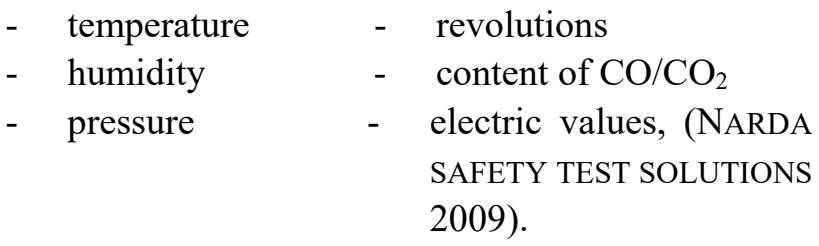

The advantage of these devices is direct reading of the measuring data from a display.

\section{Conclusion}

The integrated "Laboratory of the Objectivisation and Evaluation of Environmental Factors" is situated at the Mechanical Engineering Faculty, the department of Process and Environmental Engineering. The laboratory equipment and its instruments and technologies are able to measure the basic physical factors of working indoor and outdoor environments. At present there are 5 environmental factors: noise, vibration, illumination, electromagnetic radiation and micro-climate.

It is necessary to obtain more environmental educated engineers who participated in qualitative academic preparation. But technical science and development can also claim high quality IT skills. It is a challenge for academic educational institutions to build a lot of well equipped laboratories. The establishment of a "Laboratory of the Objectivization and Evaluation of Environmental Factors" undoubtedly contributes to increased quality of studies and preparation for undergraduates.

\section{Affiliation}

This article was elaborated within the Project KEGA: 074TUKE-4/2015 An innovative approach to legislative coordination of environmental protection through the visualization on the basis of the phenomenon Small World Networks.

\section{Literature}

1. Chovancová J., Harausová H. 2013: Education of the next generation of managers in context of green economy. Journal of economic development, environment and people. Vol.2.

2. MARKULIK, Š., NAGYOVÁ, A. 2008: Quality management system + university $=$ myths or obligation? In: ORP '2008. 6 p. - ISBN 8493425656

3. NORSONIC-PHONOMETERS 2009, [online-cited 4th Decem-ber]. Accessible on www.norsonic.com/ index.php?sideID $=2431$ \&ledd $1=943$

4. CRYSTAL InSTRUMENTS 2009 [online-cited 4th December]. Accessible on www.goci.com/products.asp

5. Testo 400 A 4352009 [online - cited 7th November]. Accessible on www.grimm.sk/testo400 testo $435 . \mathrm{htm}$

6. TESTO 5452009 comfort operating luxmeter [online - cited 7th November]. Accessible on www.grimm.sk/Testo545\%20-\%20Luxmeter.htm

7. NARDA SAFETY TEST SOLUTIONS 2009 [online cited 7th November]. Accessible on http://www.narda-sts.us/products lowfreq meters.php 